\title{
El docente universitario y el tiempo laboral, una mirada desde la configuración y mecanización a partir de la acción propuesta por el sistema capitalista
}

\author{
Por: Jhon Mario Marín Dávila \\ Trabajador social en formación \\ Investigador del semillero Laboratorio de Pensamientos, Naturaleza y Cultura \\ Corporación Universitaria Minuto de Dios - Sede Bello
}

\begin{abstract}
RESUMEN
La presente investigación preguntó por el tiempo social y su incorporación acelerada en la producción laboral del docente universitario. Estima diferencias entre el tiempo cronológico, la mecanización del cuerpo y la configuración mental, problematizando en la percepción del tiempo significativo del docente. Evidencia cómo se está modificando la percepción del tiempo significativo en su estilo de vida, alienándolo al tiempo cronológico manipulado por el sistema económico. En el proceso hace un análisis del tiempo, la carga laboral y de cómo el sistema capitalista crea contratos precarios para los docentes a partir de su circuito acelerado en la producción académica. Se realizó desde un enfoque cualitativo e interpretativo con una postura crítica, constructivista; nutriéndose del método etnográfico sumado a rastreos bibliográficos, grupo focal alrededor de un performance, cuadros comparativos, entrevistas y triangulación de información, entre otras. Como resultado se obtuvo que la construcción supuesta por el capitalismo les permite a los docentes estar en constante acción para lograr mayor producción, alcance de metas e indicadores en su plan de trabajo imponiéndose sobre su tiempo significativo, y que las formas contractuales e ideales administrativos de las universidades trasgreden la esencia del docente volviéndolo una máquina para mayor producción.
\end{abstract}

Palabras claves: Tiempo social; tiempo laboral; contrato laboral; sistema capitalista; universidad como empresa; mecanización, docente.

\begin{abstract}
The present investigation asked for the social time and its accelerated incorporation in the labor production of the university teacher. It estimates the differences between chronological time, the mechanization of the body and the mental configuration, problematizing the perception of significant teacher time. Evidence how it is modifying the perception of significant time in their lifestyle, alienating it to the chronological time manipulated by the economic system. Does An analysis of time and workload and Of how the capitalist system creates precarious contracts since teachers from its accelerated circuit in academic production at the university. This investigation was done from a qualitative and interpretive approach with a critical posture, constructivist; through the ethnographic method added to bibliographic traces, focus group around performance, comparative tables, interviews and triangulation of information. As a result, it was obtained that The construction supposed by capitalism enables teachers to be in constant action to achieve great production, achievement of goals and indicators in their work plan imposing themselves on their significant time and that the contractual forms and administrative ideals of the universities transgress the essence of the teacher, turning them into a machine for greater production.
\end{abstract}

Key words: Social time; work time; employment contract; capitalist system; university as a company; mechanization; teacher. 


\section{INTRODUCCIÓN}

La siguiente ponencia surge a partir de la culminación del proyecto de investigación "El tiempo laboral del docente de trabajo social en la Corporación Universitaria Minuto de Dios sede Bello, un análisis desde la mecanización de la acción propuesta por el sistema capitalista". Adscrito al grupo de investigación Trayectos y al Semillero de investigación Laboratorio de Pensamientos, Naturaleza y Cultura.

Se ejecutó la investigación para el alcance del objetivo General: Analizar la incidencia que tiene el sistema económico en la percepción de la aceleración del tiempo para la mecanización de la acción del sujeto trabajador docente de trabajo social Uniminuto sede Bello, y de los objetivos específicos: 1. Describir la percepción del tiempo social (cronológico y significativo) desde los sujetos trabajadores docentes de trabajo social Uniminuto sede Bello; 2. Comparar la influencia del tiempo cronológico y significativo en el alcance del bienestar laboral de los sujetos trabajadores docentes de trabajo social Uniminuto sede Bello y 3. Vislumbrar la percepción que tienen los docentes del programa de trabajo social de la corporación universitaria minuto de dios, sobre el tiempo y la mecanización de la acción para lograr una mayor producción y alcance de metas e indicadores en su plan de trabajo. Para así responder a la pregunta de investigación: ¿Cómo la mecanización laboral a partir del tiempo capitalista, limita la acción consciente del sujeto trabajador docente de Trabajo Social en la Corporación Universitaria Minuto de Dios sede Bello?

En un primer momento se hizo una comprensión teórico conceptual del tiempo y de los quehacer de la misión docente en un marco capitalista encontrando que la esencia del docente colombiano ha ido mutando con las dinámicas educativas - administrativas, tras un ideal de progreso y calidad para estar a la par en mundo globalizado, este "discurso de la calidad funciona, además, como un discurso moral, que apela a la responsabilidad de los actores del funcionamiento de las organizaciones, culpabilizándolos de su crisis o fracaso e induciéndolos a que sean más productivos". (Bolívar, 1999, p. 4)

En esta lógica ilusoria, las instituciones alienan al docente, le genera más producción con la excusa de que esta subirá los indicadores y estarán compitiendo con las mejores instituciones universitarias. Bajo este discurso el docente tiene más actividades, lo que empieza alejarlo de su esencia de enseñar. Ya lo diría Han (2015) "el esclavo se libera del dominio del amo, pero paga un precio: convertirse en un esclavo del trabajo". (p, 140)

Al analizar esta situación desde el tiempo sistémico, el cual se divide en tiempo cronológico y significativo:

El tiempo sistémico (o capitalista) hace referencia al tiempo cronológico o cuantitativo y el tiempo del mundo de la vida al tiempo significativo o cualitativo. Ambos tiempos coexisten entre sí y regulan la vida social lineal y significativamente al producir significados en periodos concretos de tiempo histórico. (Maldonado, 2016, p. 116)

El tiempo cronológico desde el docente es entendido como: horas labores, contratación, tareas asignadas, reuniones programadas, tiempo y horarios de dar la clase, fechas límites para entregar investigaciones, informes o resultados de proyectos, actividades sustantivas, entre otras; que le permite tener una organización en su trabajo. Y el tiempo significativo: vivencia desde su sentípensar, compartir con colegas, amigas o amigos, familiares, ratos de soledad, estudio por gusto propio, espacios académicos no obligatorios, conversas, entre otras; que permiten en su vida 
decisiones autónomas, mejor disfrute, incremento de sus relaciones naturales y humanas y mejora de su buen vivir.

Ahora bien, el sistema capitalista bajo su premisa de producción acelerada para mayor acumulación, manipula el tiempo cronológico en beneficio propio, para mecanizar y alienar a los docentes, extinguiendo lo mayor posible su tiempo significativo, el cual es fundamental para el buen vivir, la salud mental y física del docente.

Esto se evidencia en la sobre carga laboral con actividades distintas a la de enseñar, la sobrepoblación de estudiantes en un salón clase que oscila entre 35, 40 o más, 10 o más estudiantes para asesoría de trabajo de grados o prácticas profesionales, la presión de siempre estar produciendo artículos o ponencias, contratos inciertos por solo los 4 meses del semestre lo que suman en un año 4 meses sin empleo, salarios bajos acordes a sus estudios y horas extras que asumen desde su propio tiempo para cumplir con los productos.

\section{METODOLOGÍA}

La presente investigación se desarrolló desde lo cualitativo, se nutrió desde el método etnográfico y se caracterizó por ser interpretativa desde una postura crítica y constructivista. Realizando desde una visión temporal un análisis de la situación laboral del docente universitario, el cual devela la precariedad de su trabajo, mecanización, plusvalía y alienación con la institución universitaria.

\section{MOMENTOS DE LA INVESTIGACIÓN:}

1. Realización de un rastreo bibliográfico de investigaciones, artículos o libros en revistas indexadas, bibliotecas, trabajos de grado, tesis de maestría y doctorado elaborados entre el 2010 y el 2019 que permita reconocer información sobre el tiempo social, tiempo acelerado y cómo estos afectan al sujeto trabajador. Fichándose en un cuadro de Excel con autor, publicación, cita textual y palabras claves, por categorías, triangulándose con las actividades de campo e investigación con los docentes.

2. Implementación de un grupo focal con los docentes, donde se discutió sobre el tiempo social a partir de la percepción generada tras la presentación del performance "La aceleración del tiempo: mecanización del docente, por la acción propuesta del tiempo capitalista".

3. Se ejecutó una entrevista etnográfíca con 13 docentes universitarios, que permitió generar insumos de cómo estos perciben el tiempo cronológico, el tiempo significativo y cómo esto influye es su vida cotidiana, laboral y para su bienestar. Caracterizándose dichas observaciones en un cuadro de Excel y triangulándose la información por métodos deductivos. (Se lograron realizar solo 13 etnografías siendo pensada para 16, unas etnografías se realizaron por video llamada desde la entrevista. Esto por las dinámicas generadas del aislamiento a partir de la emergencia social y sanitaria de la COVID-19).

5. Se desarrolló un cuadro comparativo que permitió diferenciar el tiempo cronológico y significativo que viven los docentes, teniendo en cuenta los insumos de los instrumentos aplicados anteriormente. En Excel se creó un cuadro dividido entre información del tiempo cronológico e información del tiempo significativo sumado a una tercera celda para el análisis. 
6. Interpretación de los resultados, desde una triangulación de toda la información obtenida. Con la base de datos en Excel donde se sistematizo cada instrumento aplicado y se codifico según la categoría de análisis a la que se deba respuesta. Luego se comparó la teoría con la respuesta y se buscó una linealidad de reflexión, critica o rechazo a los postulados categoriales. Dando respuesta con conclusiones a las diferentes variables.

\section{RESULTADOS}

\section{TIEMPO CAPITALISTA EN LAS INSTITUCIONES UNIVERSITARIAS}

El tiempo capitalista (cronológico), tiene como objetivo desde su lógica de producción educar cuerpos mecánicos que creen riquezas y sigan beneficiando a unos cuantos. En el caso de las instituciones universitarias en el objetivo de mejoras indicadores y alcances de metas para tener mayor reconocimiento y acreditación; acelera la producción, genera en el cuerpo del docente universitario una mecanización y lo sumerge en un círculo que no tiene salida: de la casa a la universidad y de la universidad a la casa. Provocando un desgaste de energía y menos ganas de sentir y pensar.

Esta acción repetitiva en labores y qué hacer personal del docente, lo llevan a una mecanización de una misma acción, logrando un control de su tiempo, educación de su cuerpo y cerebro. Desde una analogía pasa a verse como una máquina, como un engranaje que solo desarrolla una función a la velocidad que se le exige y se limita a obedecer la lógica de producción de la institución. Han (2015). Manifiesta que "El cálculo puro como trabajo está estructurado por una temporalidad que no deja lugar a la demora. Desde la óptica del procedere, la demora solo sería una paralización que debería eliminarse lo antes posible”. (p. 104).

Parafraseando a Marx (1976) el sistema económico capitalista se transforma con el trasegar de la historia y continúa prevaleciendo su objetivo, con la acumulación de riqueza y circulo de producción. Teniendo como base la mano de obra como fuente principal para lograr la producción; con apoyo de las máquinas y en la actualidad con la especialización del conocimiento con tareas al sujeto en el trabajo.

Este sistema desde el control y forma para producir riqueza, acelera la producción y no solo en las empresas o fabricas sino también en las instituciones educativas como se menciona en los párrafos anteriores. Crea una pérdida de conciencia en el espacio-tiempo del sujeto docente, puesto que, se rige bajo unas jornadas intensivas que debe cumplir para generar una cantidad de producción y cumplimiento con unos requerimientos de absoluta obligatoriedad, como: actividades sustantivas, dictar clases, reuniones, asesorías, realización de informes, investigaciones, manejo de aulas virtuales, capacitaciones, aprendizaje de otro idioma, entre otras. "Ya no trabajamos para nuestras necesidades, sino para el capital. El capital genera sus propias necesidades, que nosotros, de forma errónea, percibimos como propias”. (Han, 2014, p. 10).

En este sentido, a medida que el docente acelera la percepción del tiempo se va normalizando, pasando a un tiempo "normal". Por esto, el docente se pregunta ¿estoy cansado? Siendo el mismo tiempo laboral, "los relojes establecen un orden duradero, pero no simbolizan la 
aceleración del tiempo". (Davila, 2016, p. 61). Obviando, que no es acelerado el tiempo, este es el mismo, sino que con todas las labores que ejerce para cumplir con la institución en el tiempo pactado las hace a un ritmo rápido, teniendo un mayor desgaste físico y espiritual. En otras palabras, este es "un modelo de producción capitalista que busca el máximo beneficio y rendimiento en un menor tiempo" (Maldonado, 2015b, p. 123).

Parafraseando a Marín y Chaves (2019) el docente empieza a cambiar su vida, deja de tener poder de decisión, pierde autonomía a la hora de hacer el trabajo, opaca su pensamiento analítico, ético, político y crítico y se sumerge en una alienación donde su voz calla en las situaciones que no está de acuerdo, así, termina por obedecer en todo lo que le dicen y se autodisciplina.

Cuando el docente manifiesta estas situaciones y malestar por el exceso de plusvalía, entiendo esta como: "La plusvalía producida mediante la prolongación de la jornada de trabajo es la plusvalía absoluta, mientras que la que se logra reduciendo el tiempo de trabajo necesario, con el incremento de la productividad, es la plusvalía relativa". (Ahumada, 2006, p.385), para recuperar su tiempo propio, tiempo libre en el cual pueda descansar, no sobre esforzarse, vivir, sentir, pensar, tener espacios de ocio y con la familia. Las instituciones dicen: las puertas están abiertas o argumenta que, sino les basta con el horario flexible, benéficos institucionales, incentivos por publicar y producir, entre otras. Respuestas que degradan más el trabajo docente, haciéndolo perder esperanza de vida, la creatividad, el pensar y el sentir. En otras palabras:

La identidad del maestro se aleja cada vez más de la primera esencia de la universidad, la cual le fue conferida por su autonomía y tradición, su construcción crítica, el compromiso ético social y la libertad de enseñanza y formación; por consiguiente, el referente moral, aquel orgullo que emanaba la institución, se pierde como generador de sentido de pertenencia y lastimosamente de la identidad de sus profesores. (Cabrales, 2016, p.49)

La identidad del docente es transformada por la mutación de la educación a partir de la lógica acelerada y acumulación del tiempo capitalista, alejándolo de su esencia de enseñar y aboliendo su tiempo significativo.

\section{MECANIZACIÓN Y CONFIGURACIÓN DOCENTE}

La sobrecarga laboral del docente tiene implicaciones en su cuerpo, pues esta logra una mecanización que lo limita a unas pocas funciones repetitivas en su trabajo, por ejemplo, en el aula de clase se encuentra en un espacio-tiempo ya estipulado por la institución universitaria, donde entra y se encuentra ya todo un lugar estructurado, televisor, tablero, sillas en filas, y el espacio del frente para el desarrollar su actividad. Dentro de esta aula de clase responde por un tiempo de laboral, en el cual tiene que producir o compartir un conocimiento para transmitirlo al estudiante; la postura para dar la clase es monótona, opresiva a su cuerpo puesto que tiene que tener una postura "normal" por las horas de clase para tener más respeto por el estudiantado.

O en el caso de la oficina o facultad sitio de estudio en la facultad, está en un lugar y única posición sentado, cumple un horario estipulado en donde hace los informes, calificaciones, diferentes actividades y entre otras, donde muchas veces el tiempo no le alcanza y se lo lleva para la casa aumentando la plusvalía absoluta. 
El docente pasa hacer objeto y deja de ser sujeto, pues, este cae en un mecanismo u opresión de su cuerpo y una configuración e instrumentalización de su pensar y razonar, con la finalidad de beneficiar la institución sin importar que pasa con el pensar, sentir, razonar y tiempo significativo del docente. "Este trabajo alienante produce que el hombre mismo, su cuerpo, su espíritu y su mundo, le sean ajenos y hostiles". (Sossa, 2010, p. 51). O en palabras de Cabrales (2016) "El docente ya no forma para la vida, para la trascendencia, sino para contribuir al ensamblaje de estudiantes y ponerlos eficazmente en el mercado". ( $p, 56)$ y también competir con el fin de posicionar la institución universitaria en los primeros puestos de las clasificaciones académicas.

Por esto se direcciona la educación solo para la producción y replicaciones, mas no para el razonar, pensar y sentir. Pero, la crítica no recae sobre el docente, porque esta permeados bajo las lógicas de este sistema predominante; la crítica es a la poca reflexión sobre esta problemática en los docentes, que lleva silenciosamente a una castración del pensamiento, sentir y razón en la educación y si esta castración se materializa se seguirá hablando metafóricamente del docente como un "robots".

\section{CONDICIONES LABORES DOCENTE}

Las condiciones laborales del docente son paupérrimas, carecen de empatía y dignidad, en las palabras anteriores se evidencia una síntesis de cómo se explota y existe la plusvalía en el docente a partir de la sobre carga laboral. Continuaremos con una mirada de la contratación actual y de cómo la institución universitaria funciona más como una empresa que como universidad.

En la docencia estos son los contratos más utilizado: contratación a tiempo completo 48 horas semanales, medio tiempo 24 horas semanales y catedra 12 horas semanales, el docente manifiesta que en cualquiera de los 3 contratos hay que hacer horas extras para cumplir con las labores pactadas con la institución porque de lo contrario no cumplirían y estaría en peligro su trabajo.

Los 3 contratos tienen en común que pueden ser a término definido, indefinido o por prestación de servicios, la gran mayoría de docentes tiene contrato por los 4 meses que dura el semestre académico, en las vacacione universitarias se quedan sin trabajo y solo les avisan si van hacer contratados una semana antes de un nuevo semestre académico.

El docente vive en una incertidumbre si será contratado o no cada semestre, esto sumándole a los 4 meses en el año que no esta contratado por la institución universitaria, a lo que lo obliga gastarse su liquidación para sobrevivir esos meses o a mantener uno, dos trabajos o un emprendimiento para poder suplir sus necesidades. Sumado a esto manifiesta que el salario no es acorde para todo el trabajo que tiene que hacer y la inversión en estudios que tiene que hacer para cumplir un perfil y ser contratado.

El docente con estas condiciones precarias, se limita a no a cuestionar y analizar acerca de lo económico y lo político de su trabajar, para poder suplir sus necesidades y mantener su puesto asegurado, puesto que la competencia laboral es muy grande. En palabras de Cabrales (2016). "el trabajo de los profesores se encuentra determinado por la atención a los clientes de la industria de la educación, en el que deben competir con otros profesionales para conseguir una remuneración adecuada". (p. 36) 
Bajo esta lógica, las instituciones comercializan una idea: entre más productivo el docente, tendrá una vida más feliz, sin importar las condiciones básicas, a sabiendas que es una artimaña porque:

La consigna es producir en función de la innovación y la innovación en función del mercado donde hay que cumplir con indicadores para que el producto sea comercializado. Hay una aceleración del tiempo en función de la producción académica que no indica aceleración de la producción académica estratégica, así como tampoco indica que a mayor productividad mayor sea la solución a los problemas vitales de la sociedad. (Maldonado, 2015a, p. 44)

Una argumentación con un sustento egoísta y de deterioro, ya que la aceleración en la producción solo beneficia a unos pocos, pero perjudica a un gran número de docentes, se hace necesario hacer las pausa y mirar detenidamente todas estas situaciones que hacen perder la esencia del docente por la mecanización de la acción propuesta del sistema capitalista.

\section{CONCLUSIONES}

Las instituciones universitarias regidas bajo el sistema capitalista, en su lógica de producción y acumulación acelerada para mayor producción y reconocimiento en el estatus universitario, ve al docente como un objeto que explota al máximo, dejándolo de ver como un sujeto que siente, piensa y razona.

El docente tras las acciones repetitivas que realiza por su sobrecarga laboral, se sumerge en un círculo que no tiene salida y siempre lleva al mismo lugar, trayendo como consecuencias la mecanización de su cuerpo, configuración mental, perdida del tiempo significativo y problemas físico y espirituales.

La universidad paso hacer dominada por los intereses mercantilistas convirtiéndose en una empresa que no le importa la condición laboral de los docentes, importándole más el estatus universitario, metas e indicadores y la cantidad de productos, que la calidad y el respeto por la creación de conocimiento.

A partir de las condiciones labores precarias que mecanizan al docente y configuran su mente, la enseñanza, creación de conocimientos, el amor por los procesos pedagógicos va en declive a una instrumentalización de unos intereses políticos y económicos gobernados por el sistema capitalista, con la finalidad de tener mayor de mano obra obediente y productora de ganancias y no seres sentípensantes que no generen ganancias significativas.

El docente se encuentra inconforme, pero, las necesidades económicas para sobrevivir en la sociedad y el discurso de amar lo que se hace, sin importar los esfuerzos y ser agradecidos por estar mejor que otros; hacen opacar el análisis crítico y tener una postura contradictoria a su pensar, en cuanto a sus condiciones laborales, alienaciones y la plusvalía absoluta y relativa que viven. 


\section{BIBLIOGRAFÍA}

Ahumada, M. C. (2006). La teoría marxista de la plusvalía absoluta: una clave para entender las condiciones laborales en el periodo neoliberal. En: Marx Vive 2004, Marx Vive: teoría y acción política en el capitalismo actual. Bogotá: Universidad Nacional de Colombia, 2006. v. 1, 355-388.

Bolívar, A. (1999). La educación no es un mercado. Crítica de la "Gestión de Calidad Total". Aula de innovación educativa, 83-84, pp. 77-82.

Cabrales, O. (2016). Trayectorias laborales y perspectivas sobre el trabajo docente universitario en Colombia. Bogotá, Colombia: Universidad Militar Nueva Granada.

Dávila, E. (2016). Rápido a ninguna parte. Consideración en torno a la aceleración del tiempo social. Acta sociología, (69), 55-75.

Han, B. (2014). Psicopolítica: neoliberalismo y nuevas técnicas de poder. Barcelona: Herder Editorial.

Han, B. (2015). El aroma del tiempo: un ensayo filosófico sobre el arte. Barcelona: Herder Editorial.

Marx, K., (1976). El capital. Critica de la economía política, libro 1 - tomo 2. Ediciones Akal, S.A. España.

Maldonado, C. E. (2015a). El progreso como tiempo mítico secularizado. quaest.disput, Vol. 8 (16), 28- 53.

Maldonado, C. E. (2015b). El tiempo social contemporáneo desde una perspectiva acelerada. Revista de Antropología y Sociología: VIRAJES, 17(2), 121-134.

Maldonado, C. E. (2016). Contradicciones del sistema y mundo de la vida desde un tiempo acelerado. Revista Colombiana de Ciencias Sociales, 7(1), 114-130.

Marín, J. M. \& Chaves, D. A. (2019). Miradas a la mecanización del Tiempo Social. Revista Subjetividades \& Sociedad: Interdisciplinariedad en las Ciencias Humanas \& Sociales, (5), 22-24.

Sossa, A. (2010). LA ALIENACIÓN EN MARX: EL CUERPO COMO DIMENSIÓN DE UTILIDAD. Revista de Ciencias Sociales (Cl), (25),37-55. 